
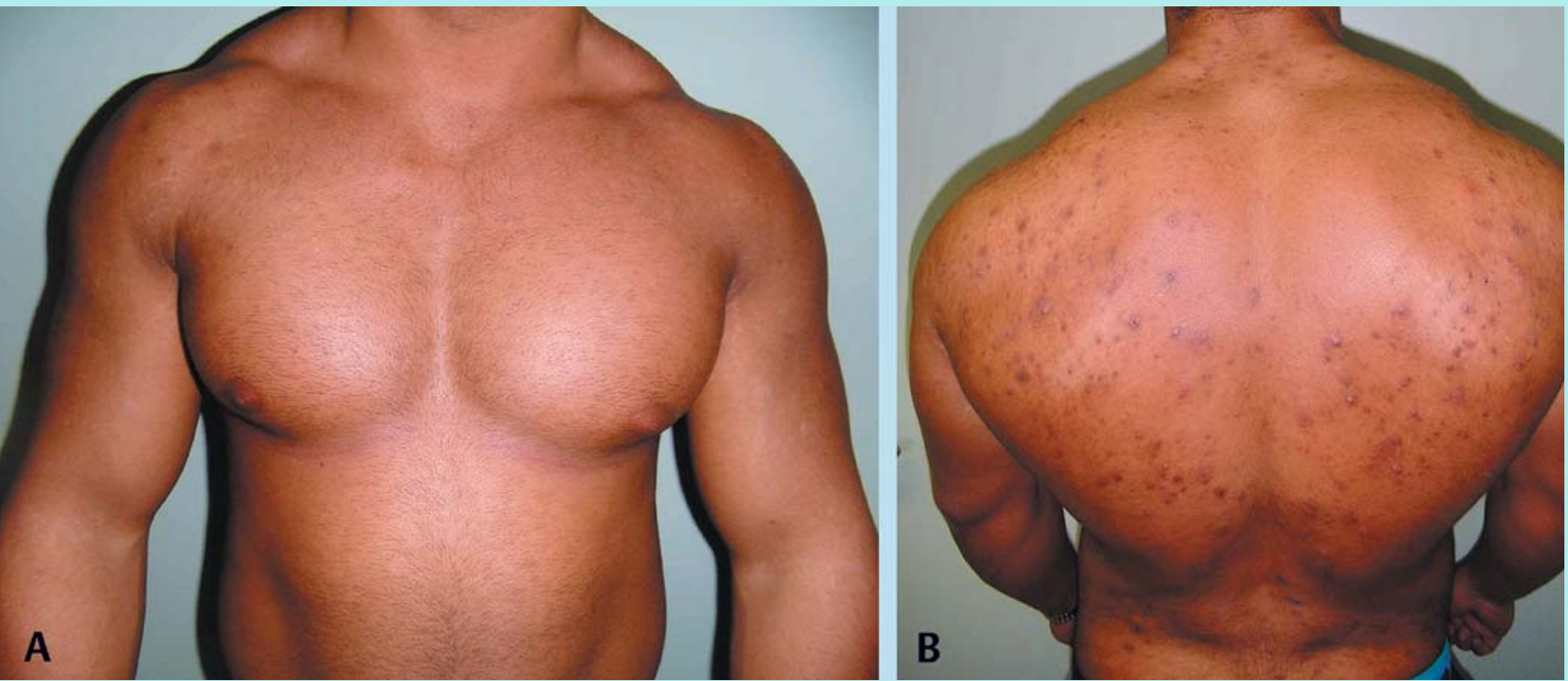

Abb.1 Vorder- (A) und Rückansicht (B) des Patienten.

\section{S. Fuchs}

${ }^{1}$ Medizinische Fakultät, Sektion Allgemeinmedizin, Martin-LutherUniversität Halle-Wittenberg

Bibliografie

DOI 10.1055/s-0034-1370099 Dtsch Med Wochenschr 2014;

139: 1299-1300 - (c) Georg

Thieme Verlag KG · Stuttgart .

New York · ISSN 0012-0472

\section{Korrespondenz}

Stephan Fuchs

Martin-Luther-Universität

Halle-Wittenberg,

Medizinische Fakultät

Magdeburger Straße 8

06112 Halle (Saale)

eMail Stephan.Fuchs@medi-

zin.uni-halle.de
In einer hausärztlichen Sprechstunde stellt sich ein 23-jähriger Mann mit seit wenigen Wochen bestehenden und störenden Hautveränderungen im Rückenbereich vor. Weitere Beschwerden werden nicht angegeben.

Die klinische Untersuchung zeigt vier pathologische Befunde.

Welche sind das?

Erlauben diese Befunde eine Diagnose?

Wenn ja, welche?

Sind Differenzialdiagnosen möglich?

Wenn ja, welche? 


\section{3-jähriger Kraftsportler mit neu aufgetretenen}
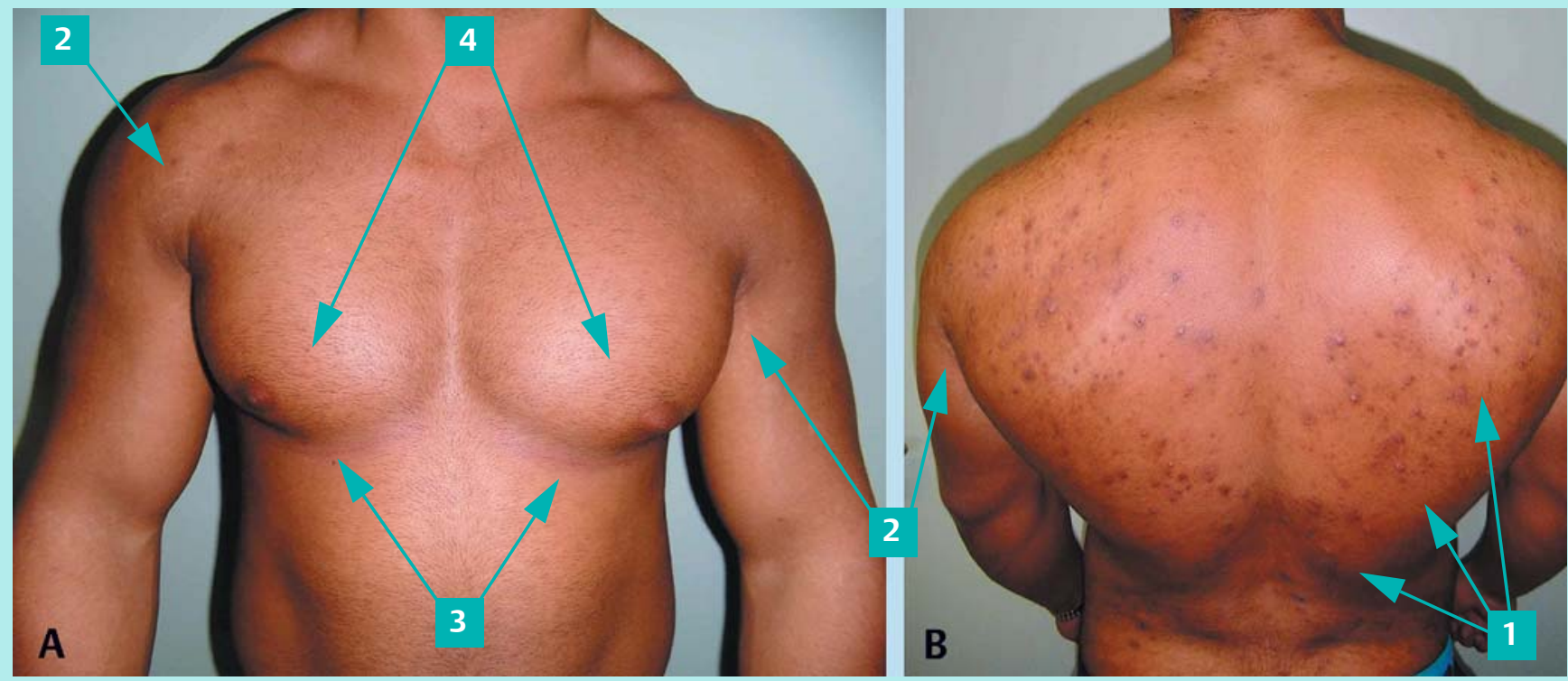

Abb.2 Vorder- (A) und Rückansicht (B) des Patienten.

\section{Befunde}

1. eruptive Papeln mit Schwerpunkt im seitlichen oberen Rücken und unteren Rücken

2. Striae cutis atrophicae (vordere und hintere Axillarregion sowie an medialen Oberarmen) als Zeichen älterer Dehnungsstreifen

3. subpectorale Striae rubrae als Zeichen neuerer Dehnungsstreifen

4. linke Pectoralis-Region voluminöser als rechte Pectoralis-Region.

\section{Diagnose}

- Testosteron-Missbrauch mit schnellem Anstieg der Muskelmasse, Steroidakne und Gynäkomastie (links > rechts)

\section{- Differenzialdiagnosen}

- endokrin aktive Hodentumoren

- androgenproduzierendes Nebennierenkarzinom

\section{Erläuterung}

Auf intensive Nachfrage berichtet der Kraftsportler über einen kontinuierlichen und mehrmonatigen Testosteron-Konsum etwa 2-3 $\times$ wöchentlich. Dieses injiziert sich der Patient abwechselnd in beide ventralen Oberschenkel. Abheilende Injektionsstellen sind erkennbar. Das Medikament bestellt der Patient über das Internet, das sterile Injektionsbesteck kauft er in regionalen Apotheken. Zusätzlich berichtet der Patient über Erektionsprobleme, einen seit mehreren Monaten unerfüllten Kinderwunsch sowie über dissoziales Verhalten.

Die Befunde stimmen mit dem geschilderten Testosteron-Konsummuster überein. Mit dem Patient wurde über die Ursache der eruptiven Hautveränderungen sowie den jetzt zusätzlich entdeckten weiteren Testosteron-Nebenwirkungen gesprochen (Androgen-induzierte Gynäkomastie, Striae unterschiedlichen Alters, verkleinerte Hoden).
Nach telefonischer Rücksprache mit einer internistischen Krankenhaus-Ambulanz wurde der Testosteron-Konsum ohne Ausschleich-Schema abrupt beendet. Zusätzlich wurde eine stationäre Aufnahme zur komplexen interdisziplinären Abklärung einer tastbaren tumorösen Veränderung der linken Mamma, urologischer Diagnostik, Ausschluss einer Anabolika-induzierten Kardiomyopathie und Evaluation der in der Fremdanamnese berichteten Verhaltensänderungen vereinbart.

Die Beendigung des Anabolika-Konsums führte zur Besserung der geschilderten Befunde. Die genannten Testosteron-Nebenwirkungen sowie der Zusammenhang mit einem Anabolika-Konsum sind bei zahlreichen Patienten beschrieben [1].

Literatur

1 Wollina U, Pabst F, Schönlebe J et al. Side-effects of topical androgenic and anabolic substances and steroids. A short review. Acta Dermatovenerol Alp Panonica Adriat 2007; 16: $117-122$ 